\title{
Neutrino predictions from choked GRBs and comparison with the observed cosmic neutrino flux
}

\author{
Irene Di Palma, ${ }^{a, b, *}$ Silvia Celli, ${ }^{a, b}$ Angela Zegarelli, ${ }^{a, b}$ Antonio Capone, ${ }^{a, b}$ Michela \\ Fasano $^{a}$ and Dafne Guetta ${ }^{c}$ \\ ${ }^{a}$ Dipartimento di Fisica dell'Università La Sapienza, P. le Aldo Moro 2, I-00185 Rome, Italy \\ ${ }^{b}$ Istituto Nazionale di Fisica Nucleare, Sezione di Roma, P. le Aldo Moro 2, I-00185 Rome, Italy \\ ${ }^{c}$ ORT-Braude College, Carmiel, Israel \\ E-mail: irene.dipalma@roma1.infn.it, silvia.celli@roma1.infn.it, \\ antonio.capone@roma1.infn.it, michelafasano5@gmail.com, \\ dafneguetta@braude.ac.il, angela.zegarelli@romal.infn.it
}

The lack of spatial anisotropies in the sky map distribution of observed cosmic neutrinos hints towards the extra-galactic nature of their major sources. However, strong constraints are imposed by the Fermi-LAT data on the isotropic gamma-ray background in hadronic production scenarios, suggesting that the observed neutrinos might possibly originate in sources that remain hidden to gamma-ray observations. We discuss the possibility that neutrinos may come from choked jets, namely those resulting from a supernova explosion such that the collimated material fail to break out of the stellar envelope. In particular, we estimate the neutrino flux and spectrum expected from choked Gamma-Ray Bursts, focusing on Type II SNe, by performing detailed calculations of $\mathrm{p} \gamma$ interactions and accounting for all the neutrino production channels and scattering angles. We provide predictions of expected event rates for operating neutrino telescopes, as ANTARES and IceCube, as well as for under construction telescopes, as KM3NeT. We also compute the contribution of the choked GRB population to the diffuse astrophysical neutrino flux, thus providing constraints on the local rate of this source population as to reproduce the observed neutrino flux.

$37^{\text {th }}$ International Cosmic Ray Conference (ICRC 2021)

July 12 th - 23rd, 2021

Online - Berlin, Germany

\footnotetext{
${ }^{*}$ Presenter
} 


\section{Introduction}

Choked Gamma-Ray Bursts (GRB) are believed to be originated from core collapse of massive stars resulting in a relativistic fireball jet, which is unable to break through the stellar envelope [1]. A choked jet deposits all its energy in a cocoon, that eventually breaks out from the star releasing energetic material at sub-relativistic velocities. Such fast moving material has a unique signature that can be detected in early time supernova spectra [2]. However, to date, little is known concerning choked GRBs. There have been many investigations in the literature about their progenitor nature. A possibility sometimes considered in the literature is that jets might be choked in the stellar envelope of red/blue supergiant stars, hence associated with Type II SNe [3]. In this case the duration of the central engine could be longer than for typical long GRBs [4]: this is the progenitor scenario that we will consider in the following.

Neutrino emissions from choked GRBs have attracted much attention [3, 5-7], because of the possibility of explaining the IceCube diffuse flux [8] without incurring into inconsistencies with the isotropic diffuse gamma-ray background (IGRB). In this contribution we perform a Monte Carlo (MC) simulation of the $\mathrm{p} \gamma$ interactions taking place in the choked jet, from which neutrinos arise. In Sec. 2 we present the core structure of our simulation, that we adopt in Sec. 3 to estimate the expected number of neutrino-induced events in Cherenkov telescopes from a single source. Finally, we obtain the diffuse neutrino flux arising from a population of choked sources [9], and derive the expected source rate by comparing such predictions to the observed astrophysical flux.

\section{Photo-meson production}

Estimates of neutrino fluxes from astrophysical sources as a result of the $p \gamma$ interaction have been performed in the past both with analytical $[10,11]$ and numerical calculations [12-16], but no simulation for choked GRBs sources has been attempted so far. To study the expected neutrino flux on Earth from such $v$ bright (while $\gamma$ dark) sources, we have developed a MC code to simulate the particles kinematics and interactions inside the jet, allowing for a detailed description of the microphysics involved in the photo-meson process. Once the particle spectra at the source are obtained, our code calculates propagation through cosmic distances from the source to Earth, allowing a reliable estimate of the number of events expected in current and future neutrino telescopes.

In our work [9] we consider a jet propagating inside the progenitor source and define $t_{\text {jet }}$ as the jet lifetime. Such a parameter strongly depends on the physical process which powers the jet and we consider it as a free parameter of our model, possibly constrained by e.g. neutrino observations. While the jet is making its way through the star, it can be slowed down in a termination shock to a Lorentz factor much lower than its original value. If the jet crossing time $t_{\text {cross }}$ is longer than $t_{\text {jet }}$, then the jet will result choked inside the stellar envelope, hence it will not be able to break through the star surface. Specifically, we consider type II SNe as a reference progenitor scenario, by adopting within our simulation the following benchmark values characterizing the jet features: Lorentz factor $\Gamma=100$, lifetime $t_{\text {jet }}=10^{3} \mathrm{~s}$ and isotropic luminosity $L_{\text {iso }}=10^{50} \mathrm{erg} / \mathrm{s}$, where the two latter values provide a GRB isotropic energy given by $E_{\text {iso }}=t_{\text {jet }} \times L_{\text {iso }}=10^{53} \mathrm{erg}$.

Internal shocks (IS) are expected to develop in the jet interior, inward of the termination shock. Depending on the initial opening angle of the jet and the properties of the environment where it 
propagates, the jet can result in a conical shape or in a cylindrical one. While the collimation shock is most likely radiation dominated, this is not the case for internal shocks that are instead collisionless, and can hence accelerate protons and electrons to $\gtrsim 10^{5} \mathrm{GeV}$ [1].Therefore, efficient acceleration of protons is expected to be realized at the internal shocks.

We assume a first order Fermi process for primary charged particles, producing a characteristic power-law differential energy distribution of protons as:

$$
\frac{d N_{p}}{d E_{p}}=k_{\mathrm{MC}} E_{p}^{-2},
$$

where $k_{\mathrm{MC}}$ is a normalization factor resulting from the total energetics channeled into accelerated protons. Electrons are expected to lose all their energy into synchrotron radiation. However, because of the large optical thickness due to Thomson scattering, these photons thermalize in the reverse shock region (RS) to a temperature $T_{\gamma}$ such that [17]:

$$
k T_{\gamma} \simeq 313 \mathrm{eV} \mathrm{L} \text { iso, } 50 \epsilon_{\mathrm{e},-1}^{1 / 4} \mathrm{t}_{\mathrm{jet}, 3}^{-1 / 4} \rho_{\mathrm{H},-7}^{1 / 8},
$$

$k$ being the Boltzmann constant, and $\epsilon_{e}$ the fraction of $E_{\text {iso }}$ deposited into electrons. For the fraction of energy channeled into electrons and magnetic field we consider the equipartition condition $\epsilon_{\mathrm{e}}=\epsilon_{\mathrm{b}}=0.1 E_{\text {iso. }}$.

Inside the RS, the photon spectrum is well represented by a blackbody distribution, reading as:

$$
\frac{d N_{\gamma}}{d E_{\gamma}}=\frac{8}{19\left(k T_{\gamma}\right)^{3}} \frac{E_{\gamma}^{2}}{e^{\frac{E_{\gamma}}{k T_{\gamma}}}-1} .
$$

with a peak in the energy distribution given by the Wien's displacement law

$$
E_{\gamma, \mathrm{RS}}^{\max } \simeq 2.82 k T_{\gamma} \sim 881 \mathrm{eV},
$$

and a number density following the Planck distribution

$$
n_{\gamma, \mathrm{RS}}=\int_{0}^{\infty} \frac{d N_{\gamma}}{d E_{\gamma}} d E_{\gamma}=16 \pi \xi(3)\left(\frac{k T_{\gamma}}{h c}\right)^{3} \simeq 19.2 \pi\left(\frac{k T_{\gamma}}{h c}\right)^{3},
$$

where $\xi(3) \simeq 1.202$ is the Riemann zeta function. A fraction of thermal photons will then escape in the IS, since it is optically thin, where their peak energy becomes:

$$
E_{\gamma, \mathrm{IS}}^{\max }=\Gamma_{\mathrm{IR}} E_{\gamma, \mathrm{RS}}^{\max } \sim 88.1 \mathrm{keV} .
$$

where $\Gamma_{\mathrm{IR}} \sim \Gamma$ (if $\Gamma \gg 1$ [3]) is the Lorentz factor of the IS with respect to the RS. The photon density in the internal shock frame is

$$
n_{\gamma, \mathrm{IS}}=\Gamma n_{\gamma, \mathrm{RS}} f_{\mathrm{esc}} \simeq 1.9 \times 10^{20} \mathrm{~cm}^{-3} \Gamma_{2}^{2} \mathrm{~L}_{\mathrm{iso}, 50}^{-3 / 8} \mathrm{t}_{\mathrm{jet}, 3}^{-1 / 4} \epsilon_{\mathrm{e},-1}^{3 / 4} \rho_{\mathrm{H},-7}^{1 / 8} .
$$

The production of charged and neutral mesons inside a jet can take place through the resonant production of a $\Delta^{+}$by highly relativistic protons that interact with photons of the ambient radiation field, namely the thermal photons from the jet head. The algorithm simulates the photo-meson interaction for each pairs of protons and photons which satisfy the threshold condition. The 
secondary particles emerging from different interaction channels are also simulated. Their energy losses in the form of synchrotron radiation, inverse Compton scattering off the dense thermal radiation field, synchrotron self Compton and adiabatic losses are accounted for. As we will show in the following, we identify synchrotron and adiabatic losses as the main channel for muon losses, while negligible losses affect charged pions.

In order to compute the proton path inside the thermal photon gas, it is necessary to invert the path probability distribution:

$$
\mathrm{dP}\left(\mathrm{x}_{\mathrm{p}}\right)=\frac{1}{\lambda_{\mathrm{p} \gamma}} \mathrm{e}^{-\mathrm{x}_{\mathrm{p}} / \lambda_{\mathrm{p} \gamma}} \mathrm{dx}_{\mathrm{p}},
$$

where $\lambda_{p \gamma}=1 /\left(\sigma_{p \gamma} n_{\gamma}\right)$ is the interaction length. The proton path obtained through the inversion of Eq. (8) peaks at $\sim 10^{7} \mathrm{~cm}$ for $\sigma_{p \gamma}=550 \mu \mathrm{b}$ (the $\Delta^{+}$resonance peak), while for the out of resonance region value of $\sigma_{p \gamma}$, namely $\sim 120 \mu \mathrm{b}$, the proton path peaks at $\sim 10^{8} \mathrm{~cm}$. This means that a proton will interact, on average, after travelling $10^{7.5} \mathrm{~cm}$ inside the source, i.e. well within the external hydrogen envelope of the star, whose radius is $\sim 10^{13} \mathrm{~cm}$.

Defining $\epsilon_{r}$ as photon energy in the proton's rest frame, we consider the following numerical values for the boundaries between each interaction channel in our simulation [11]:

- $0.2 \mathrm{GeV} \leq \epsilon_{r}<0.5 \mathrm{GeV}$ : this energy range corresponds to the peak of the cross section, where the interaction occurs with interaction probability $P_{\text {int }}=1$ and the $\Delta^{+}$resonance is produced at rest:

$$
p+\gamma \rightarrow \Delta^{+} \rightarrow\left\{\begin{array}{l}
n+\pi^{+} \\
p+\pi^{0}
\end{array} ;\right.
$$

- $0.5 \mathrm{GeV} \leq \epsilon_{r}<2 \mathrm{GeV}$ : such interval matches the secondary peak range of the cross section, where the $\Delta^{+}$resonance is produced along with an additional pion $(\mathcal{N}$ is a nucleon, while both $\pi^{\mathrm{A}}$ and $\pi^{\mathrm{B}}$ can be neutral, positively or negatively charged pions):

$$
\begin{aligned}
& p+\gamma \rightarrow \pi^{\mathrm{A}}+\Delta^{+} \\
& \rightarrow \mathcal{N}+\pi^{\mathrm{B}} .
\end{aligned}
$$

Here, the interaction occurs with two possible values of probability: $P_{\text {int }}=0.6$ when $\epsilon_{r} \leq$ $1 \mathrm{GeV}$, otherwise the interaction probability is set equal to 0.4 ;

- $2 \mathrm{GeV} \leq \epsilon_{r} \leq 100 \mathrm{GeV}$ : this energy window corresponds to the plateau in the cross section, in which the three types of pions are simultaneously produced. This range is often referred to as multipion channel, where "..." indicates other particles created in the interaction:

$$
p+\gamma \rightarrow \pi^{0}+\pi^{+}+\pi^{-}+\ldots
$$

In this region $P_{\text {int }}=0.3$.

The pion decay products include leptons and photons:

$$
\begin{aligned}
& \pi^{+} \rightarrow \mu^{+}+v_{\mu} \rightarrow e^{+}+v_{e}+\bar{v}_{\mu}+v_{\mu} \\
& \pi^{-} \rightarrow \mu^{-}+\bar{v}_{\mu} \rightarrow e^{-}+\bar{v}_{e}+v_{\mu}+\bar{v}_{\mu} \\
& \pi^{0} \rightarrow \gamma+\gamma
\end{aligned}
$$


However, we do not further follow the high-energy photons produced, which will be absorbed in pair production processes. Instead we further track neutrinos, including flavor oscillations to Earth.

\section{Estimate of the neutrino events}

As described above, we can estimate the neutrino spectrum expected from choked GRBs, accounting for all the neutrino production channels and scattering angles. The particle spectra at the source obtained through our MC simulations are shown in Figure 1. In the the left panel we show the spectrum of $\pi^{-}$obtained from $\Delta^{+}$decay in the multi-pion decay channel, while in the right panel, we show the secondary particles from all $\Delta^{+}$decays that give rise to $\pi^{+}$.
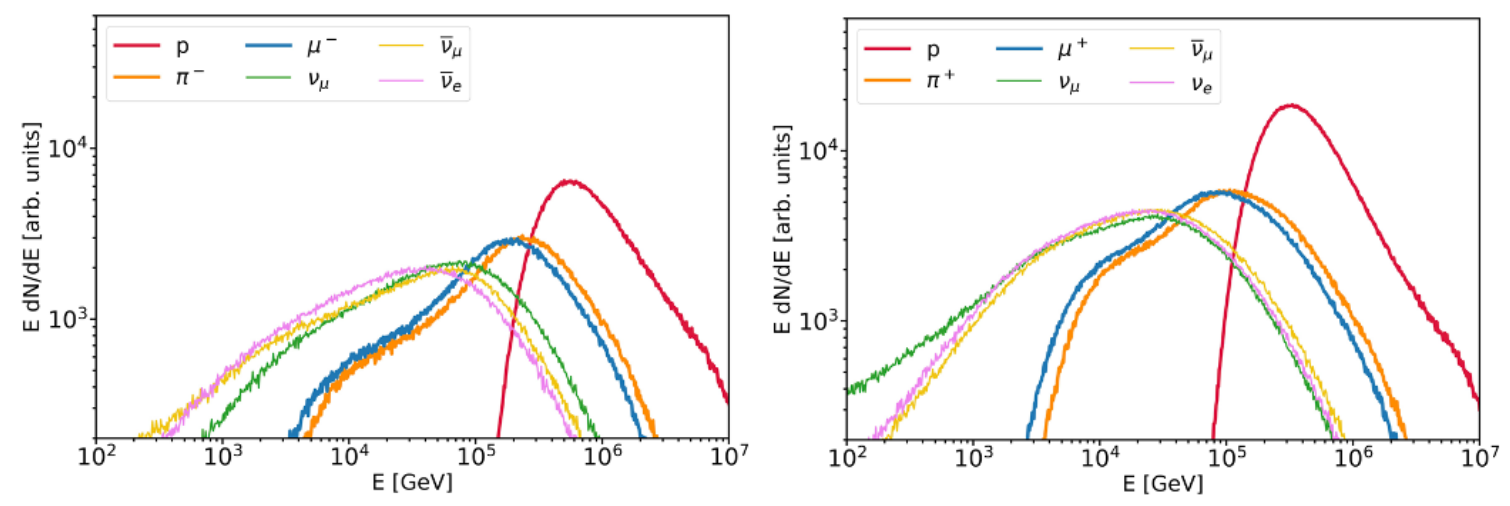

Figure 1: Particle spectra at the source arising from all interaction channels, as seen in the co-moving reference frame. Left: $\pi^{-}$channel. Right: $\pi^{+}$channel.

Transient phenomena such as GRBs are very interesting for a potential neutrino detection, since these analyses are almost background free given the well-defined space-time window provided by multi-messenger constraints. To compute the neutrino flux expected on Earth from a source at a cosmological distance, we scale the MC-obtained fluxes for three factors: the energy channeled into protons at the source, the dilution of the neutrino flux to Earth, and neutrino oscillations. We are interested in estimating the number of muon neutrino events expected from an individual source in different neutrino telescopes, since these constitute the best channel of events for astronomical studies due to their excellent angular resolution. This evaluation has been performed for ANTARES [18], KM3NeT-ARCA [19] and IceCube [20]. Note that the effective area adopted in this work for KM3NeT refers to the trigger level, being the only available information at the time of writing. In turn, the effective areas adopted for ANTARES and IceCube refer to the analysis level, as these result from detailed studies performed in the search for neutrino sources by the same Collaborations. The expected number of events per energy bin is shown as a function of the energy in Figure 2, while the total number of events expected in each neutrino telescope during the choked GRB event is given in the table. Cubic kilometer scale detectors are expected to observe $\sim 0.2$ events from such a GRB with $E_{\mathrm{p} \text {,jet }}=10^{53} \mathrm{erg}$ and $z=1$.

To estimate the expected diffuse neutrino spectrum, we assume that the rate of choked jets $R(z)$ at redshift $z$ follows the star formation rate even if there are evidences that the evolution of normal GRBs may deviate from the star formation rate $\rho(z)$, i.e. $R(z)=R_{0} \rho(z)$, where the star formation 


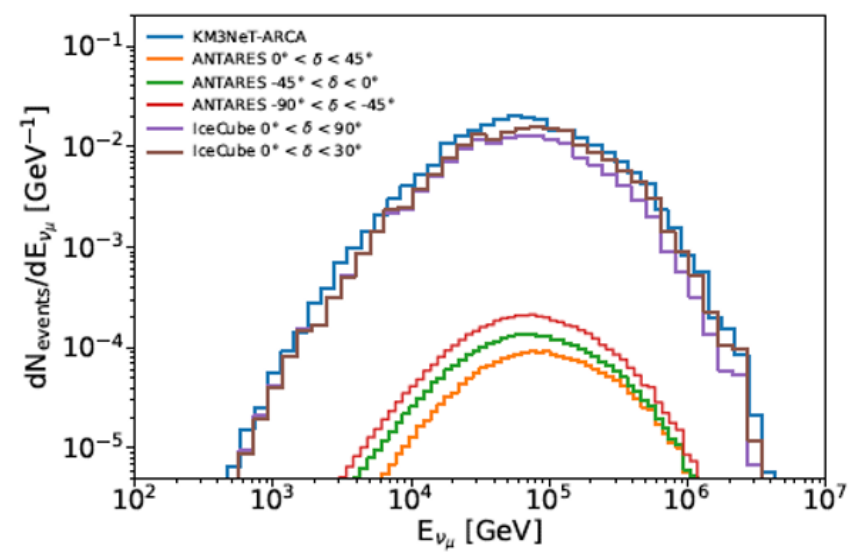

\begin{tabular}{lcc}
\hline Detector & $\delta$ & $N_{\text {events }}$ \\
\hline ANTARES & $0^{\circ}<\delta<45^{\circ}$ & $2 \times 10^{-3}$ \\
& $-45^{\circ}<\delta<0^{\circ}$ & $3 \times 10^{-3}$ \\
& $-90^{\circ}<\delta<-45^{\circ}$ & $5 \times 10^{-3}$ \\
\hline KM3NeT-ARCA & Mean $\delta$ & $2 \times 10^{-1}$ \\
\hline IceCube & $0^{\circ}<\delta<90^{\circ}$ & $1 \times 10^{-1}$ \\
& $0^{\circ}<\delta<30^{\circ}$ & $2 \times 10^{-1}$ \\
\hline
\end{tabular}

Figure 2: Left: Number of expected events in ANTARES [18], KM3NeT-ARCA [19] and IceCube [20] from a characteristic choked GRB located at different declinations. Right: Expected number of muon neutrino events in several detectors from an individual choked GRB with $L_{\text {iso }}=10^{50} \mathrm{erg} / \mathrm{s}, \mathrm{t}_{\text {jet }}=1000 \mathrm{~s}$, $E_{\mathrm{p}, \mathrm{jet}}=10^{53} \mathrm{erg}$ and $\Gamma=100$ located at different declination bands.

rate is [21]

$$
\rho(z)=\frac{(1+z)^{2.7}}{1+[(1+z) / 2.9]^{5.6}}
$$

and $R_{0}$ is the local rate of choked GRBs in $\mathrm{Gpc}^{-3} \mathrm{yr}^{-1}$. The diffuse neutrino flux is calculated via integrating the neutrino spectrum over the redshift from 0 to 8 , i.e. [22]:

$$
E_{v_{\mu}}^{\mathrm{obs}} \phi_{v_{\mu}}\left(E_{v_{\mu}}^{\mathrm{obs}}\right)=\frac{c}{4 \pi H_{0}} \int_{0}^{8} E_{v_{\mu}} \frac{d N_{v_{\mu}}}{d E_{v_{\mu}}}\left((1+z) E_{v_{\mu}}^{\mathrm{obs}}\right) \frac{\frac{\Omega}{4 \pi} R_{0} \rho(z) d z}{(1+z) \sqrt{\Omega_{\Lambda}+\Omega_{\mathrm{M}}(1+z)^{3}}}
$$

where the cosmological parameters are adopted as $H_{0}=70 \mathrm{~km} \mathrm{~s}^{-1} \mathrm{Mpc}^{-1}, \Omega_{\mathrm{M}}=0.3$, and $\Omega_{\Lambda}=0.7$, $d N_{v_{\mu}}\left(E_{v_{\mu}}\right) / d E_{v_{\mu}}$ is the differential neutrino spectrum at the source, while $\Omega$ is the solid angle of the jet, $\Omega=2 \pi(1-\cos \alpha)$, and $\alpha=0.2 \mathrm{rad}=11.5^{\circ}$ is its aperture.

In order to obtain the best value for the rate of choked GRBs, we compared the predicted flux of neutrino on Earth with the diffuse neutrino flux measured by IceCube. As experimental data we used the results obtained by IceCube both with the analysis of HESE events [23] and the parametrization of the astrophysical diffuse muon neutrino flux [24]. The flux predicted with the simulation was evaluated for several values of $R_{0}$ : the best agreement between data and our model has been obtained for $R_{0}=(1.0 \pm 0.4) \mathrm{Gpc}^{-3} \mathrm{yr}^{-1}$ (with a reduced $\chi^{2} \simeq 2.5$ ). Figure 3 shows this comparison: the central blue line represents the flux foreseen for the selected value of $R_{0}$, while the blue band represents the $68 \%$ confidence level uncertainty on $R_{0}$. In order to test the case of a less energetic choked GRB population being able to explain the observed cosmic neutrino flux, we also performed a simulation characterised by the following values of model parameters: $L_{\text {iso }}=10^{49} \mathrm{erg} / \mathrm{s}, t_{\mathrm{jet}}=200 \mathrm{~s}, \Gamma=100, t_{\mathrm{var}}=0.005 \mathrm{~s}$. Such a realization is represented by the yellow curve shown in Figure 3 for the diffuse neutrino flux contribution emerging from such a source population, which is at the level of the IceCube data provided a local rate equal to $R_{0}=(80 \pm 30) \mathrm{Gpc}^{-3} \mathrm{yr}^{-1}$. 


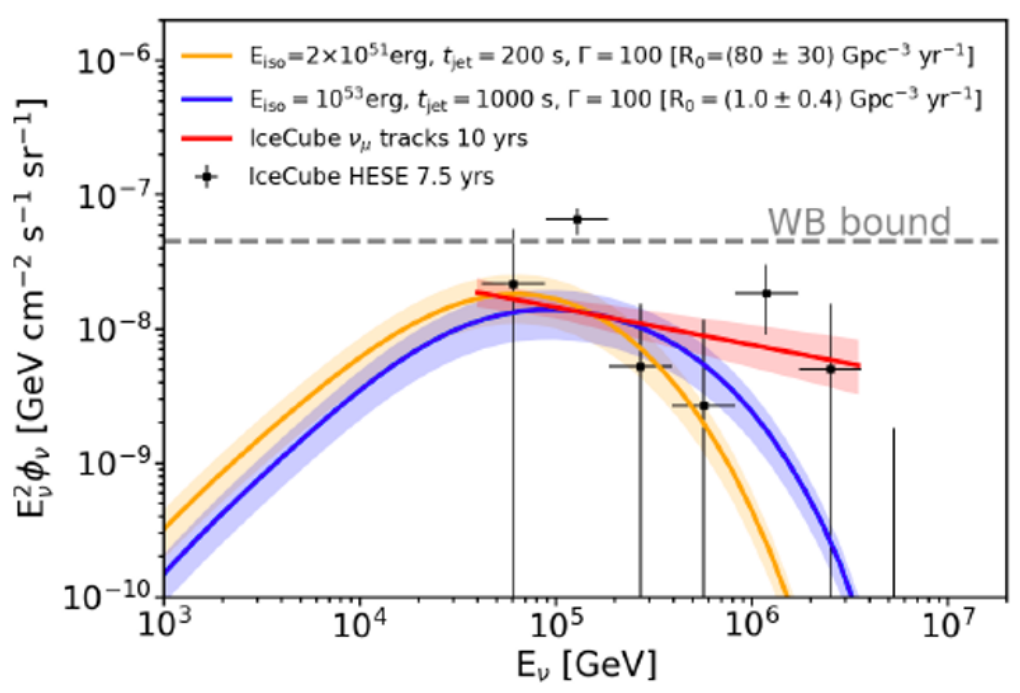

Figure 3: Diffuse neutrino spectra from choked GRBs expected on Earth, as compared with the IceCube results on the HESE data (black squares) [23], and on $v_{\mu}$ tracks (red line) [24]. The blue line refers to the GRB simulation with $L_{\text {iso }}=10^{50} \mathrm{erg} / \mathrm{s}, t_{\text {jet }}=10^{3} \mathrm{~s}$ and $\Gamma=100$ that is in better agreement with IceCube data as a function of $R_{0}$. The yellow line refers to the simulation with $L_{\text {iso }}=10^{49} \mathrm{erg} / \mathrm{s}, t_{\text {jet }}=200 \mathrm{~s}$ and $\Gamma=100$, again in better agreement with the observed cosmic neutrino flux as a function of $R_{0}$. Note that the inferred local rate of choked GRBs is consistent with the multiplet constraint for hard transient sources from [25] in the less energetic case. Additionally, the dashed grey line shows the Waxmann \& Bahcall upper bound [26]

\section{Acknowledgments}

AC, SC, IDP and AZ acknowledge the support from Sapienza Università di Roma through the grant ID RM120172AEF49A82.

\section{References}

[1] P. Meszaros and E. Waxman, TeV neutrinos from successful and choked Gamma-Ray Bursts PRL 87 (2001) 171102.

[2] T. Piran, E. Nakar, P. Mazzali and E. Pian, Relativistic Jets in Core Collapse Supernovae, ApJL 871 (2019) L5.

[3] H. He, A. Kusenko, S. Nagataki, Y. Fan and D. Wei, Neutrinos from Choked Jets Accompanied by Type-II Supernovae, ApJ 856 (2018) 119.

[4] P. Kumar, R. Narayan and J. L. Johnson, Properties of Gamma-Ray Burst Progenitor Stars, Science 321 (2008) 376K.

[5] P. B. Denton and I. Tamborra, Exploring the properties of choked Gamma-ray Bursts with IceCube's high-energy neutrinos, ApJ 855 (2018) 37.

[6] A. Esmaili and K. Murase, Constraining high-energy neutrinos from choked-jet supernovae with IceCube high-energy starting events, JCAP 12 (2018) 008. 
[7] D. Guetta, R. Rahin, I. Bartos and M. Della Valle, Constraining the fraction of core-collapse supernovae harboring choked jets with high-energy neutrinos, MNRAS 492 (2020) 843.

[8] M. G. Aartsen et al. [IceCube Collaboration], Evidence for high-energy extraterrestrial neutrinos at the icecube detector, Science 342 (2013) 1242856.

[9] M. Fasano, S. Celli, D. Guetta, A. Capone, A Zegarelli and I. Di Palma, Estimating the Neutrino Flux from Choked Gamma-Ray Bursts, arXiv:2101.03502.

[10] A. M. Atoyan and C. D. Dermer, Neutral beams from blazar jets, ApJ 586 (2003) 79.

[11] S. R. Kelner and F. A. Aharonian, Energy spectra of gamma-rays, electrons and neutrinos produced at interactions of relativistic protons with low energy radiation, Phys. Rev. D 78 (2008) 034013.

[12] K. Murase and S. Nagataki, High energy neutrino emission and neutrino background from gamma-ray bursts in the internal shock model, PRD 73 (2006) 063002.

[13] K. Murase, Prompt high-energy neutrinos from gamma-ray bursts in photospheric and synchrotron self-Compton scenarios, PRD 78 (2008) 101302.

[14] S. Hümmer, M. Rüger, F. Spanier and W. Winter, Simplified models for photohadronic interactions in cosmic accelerators, ApJ 721 (2010) 630.

[15] K. Murase and K. Ioka, TeV-PeV Neutrinos from Low-Power Gamma-Ray Burst Jets inside Stars PRL 111 (2013) 121102.

[16] N. Senno, K. Murase and P. Meszaros, Choked jets and low-luminosity gamma-ray bursts as hidden neutrino sources, PRD 93 (2016) 083003.

[17] S. Razzaque, P. Meszaros and E. Waxman, TeV neutrinos from core collapse supernovae and hypernovae, Phys. Rev. Lett. 93 (2004) 181101.

[18] S. Adrian-Martìnez et al. [ANTARES Collaboration], Search for cosmic neutrino point sources with four years of data of data from ANTARES telescope, ApJ 760 (2012) 53.

[19] S. Adrian-Martìnez et al. [KM3NeT Collaboration], KM3NeT 2.0 Letter of Intent for ARCA and ORCA, J. Phys. G: Nucl. Part. Phys. 43 (2016) 084001.

[20] M. G. Aartsen et al. [IceCube Collaboration], Searches for Extended and Point-like Neutrino Sources with Four Years of IceCube Data, ApJ 796 (2014) 2.

[21] P. Madau and M. Dickinson, Cosmic Star-Formation History, ARAA 52 (2014) 415.

[22] K. Murase and E. Waxman, Constraining high-energy cosmic neutrino sources: Implications and prospects, PRD 94 (2016) 103006.

[23] R. Abbasi et al. [IceCube Collaboration], The IceCube high-energy starting event sample: Description and flux characterization with 7.5 years of data, arXiv:2011.03545.

[24] J. Stettner, Measurement of the Diffuse Astrophysical Muon-Neutrino Spectrum with Ten Years of IceCube Data, PoS 1017 (2019).

[25] M. G. Aartsen et al. [IceCube Collaboration], Constraints on Minute-Scale Transient Astrophysical Neutrino Sources, PRL 122 (2019) 051102.

[26] J. Bahcall and E. Waxman, High Energy Astrophysical Neutrinos: the Upper Bound is Robust, Phys. Rev. D 64 (2001) 023002 [arXiv:hep-ph/9902383]. 